\title{
Is the quadrature oscillator a multivibrator?
}

\section{Lindberg, Erik}

\section{Published in:}

IEEE Circuits \& Devices

Link to article, DOI:

10.1109/MCD.2004.1364772(410) 20

Publication date:

2004

\section{Document Version}

Publisher's PDF, also known as Version of record

Link back to DTU Orbit

\section{Citation (APA):}

Lindberg, E. (2004). Is the quadrature oscillator a multivibrator? IEEE Circuits \& Devices, 20(6), 23-28. https://doi.org/10.1109/MCD.2004.1364772(410) 20

\section{General rights}

Copyright and moral rights for the publications made accessible in the public portal are retained by the authors and/or other copyright owners and it is a condition of accessing publications that users recognise and abide by the legal requirements associated with these rights.

- Users may download and print one copy of any publication from the public portal for the purpose of private study or research.

- You may not further distribute the material or use it for any profit-making activity or commercial gain

- You may freely distribute the URL identifying the publication in the public portal

If you believe that this document breaches copyright please contact us providing details, and we will remove access to the work immediately and investigate your claim. 


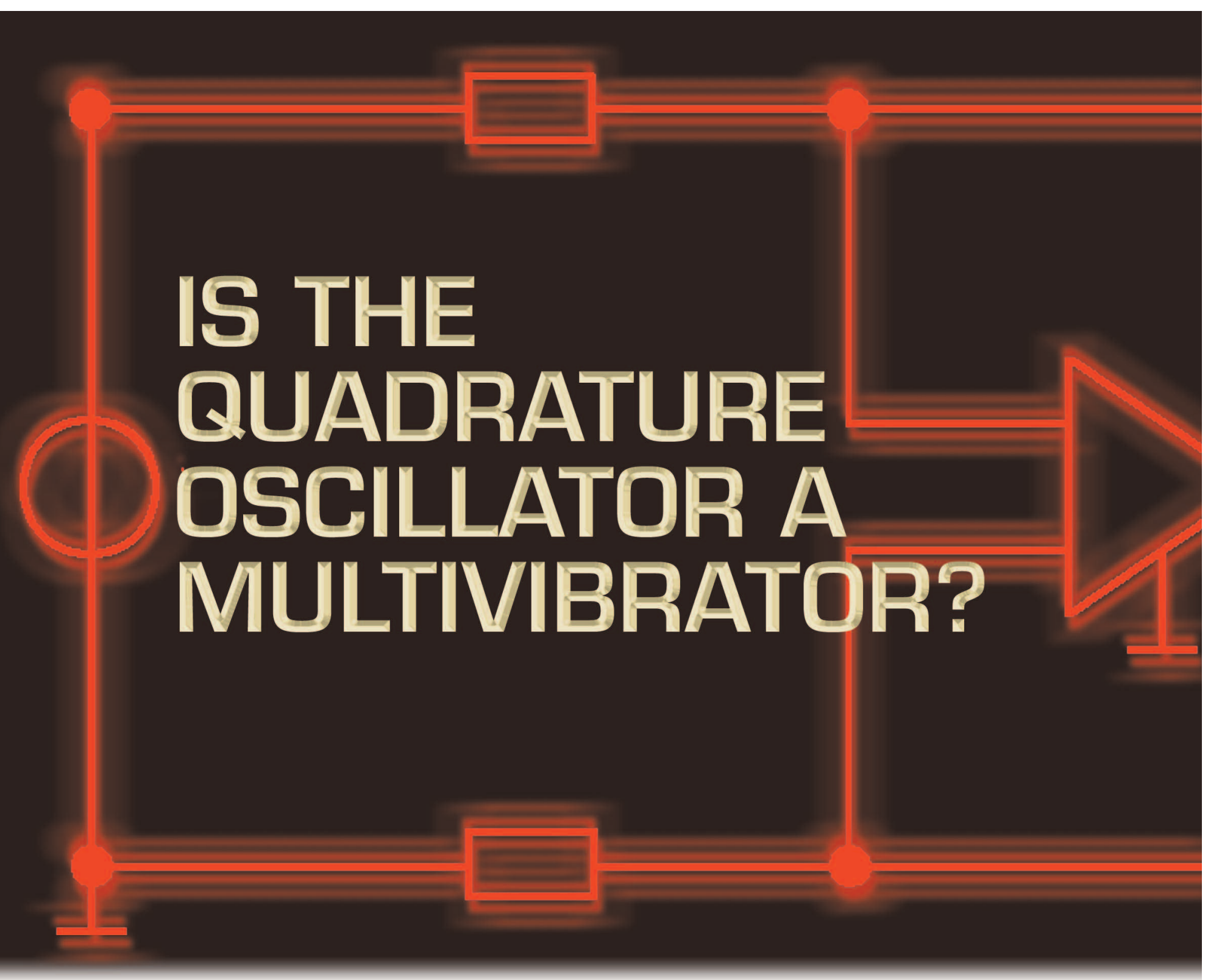

A Piecewise-Linear

Modeling Approach

to Understanding the

Mechanisms Using

"Frozen Eigenvalues"
- We aim of this article is to give insight into the mechanisms behind the behavior of oscillators from a new angle, introducing the idea of "frozen eigenvalues." This approach is based on piecewise-linear modelling and a study of the eigenvalues of the time varying linearized Jacobian of the nonlinear differential equations describing the oscillator. A multivibrator and a quadrature oscillator are used as test examples. The mechanisms behind the oscillations of the two circuits are compared.

\section{BACKGROUND}

By means of the Barkhausen Criteria, a sinusoidal oscillator is normally designed as a linear amplifier with a frequency-determining feedback circuit so that the poles of the whole circuit are on the imaginary axis of the complex frequency plane. In other words, you try to implement an ideal harmonic oscillator that may be modelled as an ideal linear inductor in parallel with an ideal linear capacitor. The ideal harmonic oscillator is mathematical fiction. It may be started up with arbitrary initial conditions and no transient time to steady-state behavior. In the real world, there are always losses to be compensated. In order to start up the oscillations, some parameters of the circuit are adjusted so that the poles (eigenvalues) of the linearized circuit are in the right-half plane (RHP) of the complex frequency plane, i.e., in the initial dc bias point, the circuit is unstable, and signals will start to increase when 
the power supply is connected. It is obvious that if the poles are very close to the imaginary axis, the time constant is very large (days, months, years?), and the transient time to steady-state behavior is very large, i.e., apparently steady-state sinusoidal oscillations take place. The placement of the poles on the imaginary axis is an impossible act of balance.

Due to the nonlinearities, the physical nature of the circuit and the signals will always be limited in some way. The crucial point is whether there will be oscillations or just a transition to a new stable dc bias point. Very little is reported about how far out in RHP the poles should be placed initially. In textbooks and papers you may find statements like: "a 'linear' oscillator is an unstable amplifier for which the nonlinearities are bringing back the initial poles in the right half plane of the complex frequency plane, RHP, to the imaginary axis"; or "a 'linear' oscillator is an amplifier with positive or regenerative feed back for which the Barkhausen condition is satisfied at a particular frequency"; or "the frequency component of the noise corresponding to the Barkhausen criteria will be amplified by the feed back circuit so that the proper sine signal builds up." This article will conclude that these statements are not true.

\section{AMPLIFIERS AND DSCILLATORS}

Amplifiers are circuits with a stable dc bias point as a reference for the signals. The differential equations used as a mathematical model for the circuit are nonlinear. The dc bias point is just the starting point at time zero. At a certain time, you may define an instant bias point and linearize the equations. For small signals, you assume that the instant $\mathrm{dc}$ bias point is time invariant and the amplifier is linear i.e. the eigenvalues of the Jacobian of the linearized differential equations are fixed in the complex frequency plane, and you may calculate the Bode plot (amplitude and phase characteristic). This assumption is called "the linear blinkers approach." For large signals, you observe distortion, and the assumption of a time invariant dc bias point is no longer valid.

Oscillators are nonlinear circuits which may have an unstable dc bias point as startup reference. If the nonlinearities are piecewise-linear, you are facing a situation where you have finite time slots where the circuit is linear, so it makes sense to investigate the eigenvalues of the Jacobian of the linearized differential equations and see whether the signals are increasing or decreasing in amplitude in a certain time slot. The trajectories

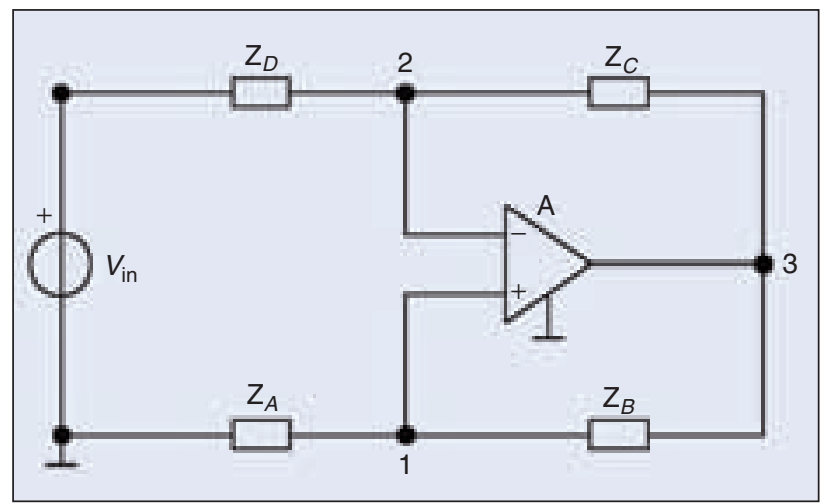

1. An amplifier with positive and negative feedback, $V(3)=A(V(1,2))$. of the eigenvalues in the complex frequency plane, the s-plane, become piecewise-linear. The eigenvalues of the Jacobian of the linearized differential equations are the poles of the network functions. It is obvious that the signals will increase in amplitude if the poles are in RHP and decrease in amplitude if the eigenvalues are in the left-half plane (LHP) of the complex frequency plane. If you assume that the poles are moving back and forth between RHP and LHP, the mechanism behind the behavior of an oscillator may be described as an act of balance between the energy you gain from the dc power supply when the poles are in RHP and the energy you loose when the poles are in LHP. In the case of a stable dc bias point, you may also observe poles in LHP all the time, and the energy is supplied as pulses in each period or half period of the oscillations.

In case of continuous nonlinearities, you may study the linearized small signal model and find the eigenvalues of the linearized Jacobian at a certain instant (the time is "frozen"). If there is only one continuous nonlinearity without break points (no jumps in the derivative) in the circuit, you may easily find the trajectories of the eigenvalues in the s-plane. If you have several nonlinearities in the circuit it becomes necessary to calculate the eigenvalues at each integration step if you want to find the trajectories in the s-plane. The study of the movement of the eigenvalues of the Jacobian of the linearized differential equations as function of time is called "the frozen eigenvalues approach" [1]-[3]. It gives you some insight in the behavior of a nonlinear circuit in the time domain similar to the Bode plot for linear circuits. Unfortunately, the concepts of amplitude and phase are not defined for a nonlinear circuit.

\section{OSCILLATORS AS UNSTABLE AMPLIFIERS}

An oscillator is a circuit for which a constant input signal (dc battery) produces an oscillating output signal (a steady-state, timevarying signal). The signal may be periodic or chaotic. An old rule of thumb says that if you want to design an oscillator, try to design an amplifier instead, and, if you want to design an amplifier, try to design an oscillator instead (i.e., invert Murphy's Law).

Figure 1 shows a perfect linear amplifier with positive and negative feedback. The input impedance of the amplifier is assumed infinite, the output impedance is assumed zero, and the gain of the amplifier is assumed constant, i.e., $V_{3}=A\left(V_{1}-V_{2}\right)$. If you introduce memory elements - capacitors, coils, hysteresis - in the four impedances, various types of oscillators may be obtained [4].

If you observe time-varying signals for zero input signal $V_{\text {in }}=0$, you have an oscillator. If the poles of the circuit are in LHP of the complex frequency plane, the signals are damped. If the poles are in RHP, the signals are undamped. The signals are steady-state signals only if the poles are on the imaginary axis. This is, of course, impossible in a real-world circuit. The ideal harmonic oscillator may be started with any initial condition and keep its amplitude and frequency constant. As mentioned above, you cannot balance on a razor's edge.

If you want to build an oscillator you must introduce nonlinearity in some way, e.g., by means of an amplifier with nonlinear gain so that for small signals the poles of the linearized circuit are in RHP and for large signals the poles are in LHP. In this case, 
you may obtain steady-state behavior based on the balance between energy you obtain from the battery when the poles are in RHP and energy you loose when the poles are in LHP. With reference to Figure 1, a Wien bridge oscillator may be designed with $Z_{A}$ as a resistor-capacitor (RC)-parallel circuit, $Z_{B}$ as a RC-series circuit, and $Z_{C}$ and $Z_{D}$ as resistors. Instead of a nonlinear gain amplifier, diodes may be introduced in $Z_{C}$ so that amplitude and frequency of the oscillator may be adjusted independently [1].

In other words, in many cases an oscillator may be considered a feedback amplifier with an unstable dc bias point. Due to the nonlinear components, the linearized small signal model corresponding to the instant bias point will vary with time. The dominating behavior of the circuit is based on the instant placement of the poles of the linearized model. If the poles are in RHP, the signals will increase in amplitude. If the poles are in LHP, the signals will diminish in amplitude.

As mentioned above, there exist stable dc bias point oscillators which are damped systems. They are based on a periodic impulse mechanism that, in the case of the pendulum clock, is called the escapement.

In the following, a common multivibrator and a quadrature oscillator are investigated by means of the frozen eigenvalues approach in order to illustrate and clarify the idea.

\section{COMMON MULTIVIBRATOR}

If you replace the impedance $Z_{D}$ in Figure 1 with a capacitor $C_{D}=0.5 \mu \mathrm{F}$ and the impedances $Z_{A}, Z_{B}$ and $Z_{C}$ with resistors $R_{A}=1 \mathrm{k} \Omega, R_{B}=1 \mathrm{k} \Omega$, and $R_{C}=2 \mathrm{k} \Omega$, you have a common multivibrator with real poles moving back and forth between RHP and LHP. The hysteresis is the second "memory component" needed for oscillations.

Figure 2 shows the voltages as functions of time using an RC4136 operational amplifier (op amp). The time constant becomes
$\tau=R C=-\left(\left(R_{A} R_{C}\right) / R_{B}\right) C_{D}=-1 \mathrm{~ms}$ for very large $A$ and $\tau=R_{C} C_{D}=+1 \mathrm{~ms}$ for $A=0$. This is in agreement with Figure 2 .

Assuming perfect an op amp with gain $A$, the pole of the circuit is $+1 \mathrm{k}$ for very large $A$ and $-1 \mathrm{k}$ for very little $A$. For $A=2$, the pole passes from $+\infty$ to $-\infty$, as shown on Figure 3. Please note that this is not a jump but a smooth transition.

\section{QUADRATURE OSCILLATOR}

Quadrature oscillators are based on a feedback loop with at least one almost ideal integrator for which input and output are two sinusoids with $90^{\circ}$ phase difference. In [5], an active $\mathrm{RC}$ integrator and a passive $\mathrm{RC}$ integrator are combined with a negative resistance. The mechanism with a complex pole pair moving between RHP and LHP may be observed.

If two amplifiers are coupled as shown in Figure 4, you may have a quadrature oscillator if the impedances are chosen as resistors according to [7]. In this case, you make use of the op amp poles as frequency, determining memory (integrating real poles) and the nonlinear saturation characteristic of the op amp as the amplitude limiting mechanism.

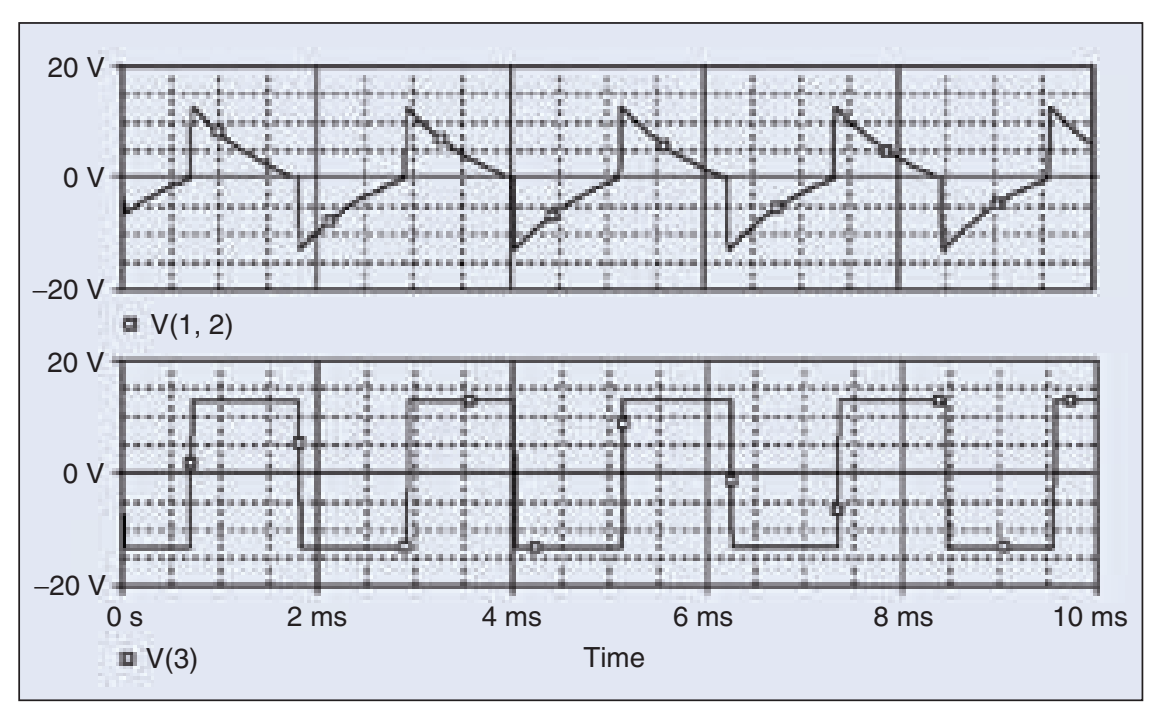

2. Common multivibrator, PSpice analysis, op amp input voltage $V(1,2)$ and op amp output voltage V(3) as functions of time.

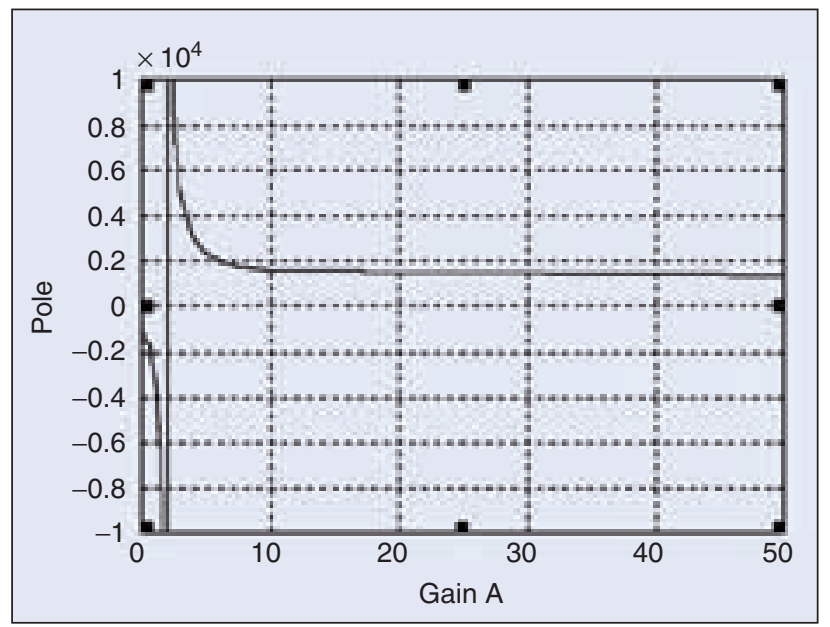

3. Multivibrator pole as function of amplifier gain $A$.

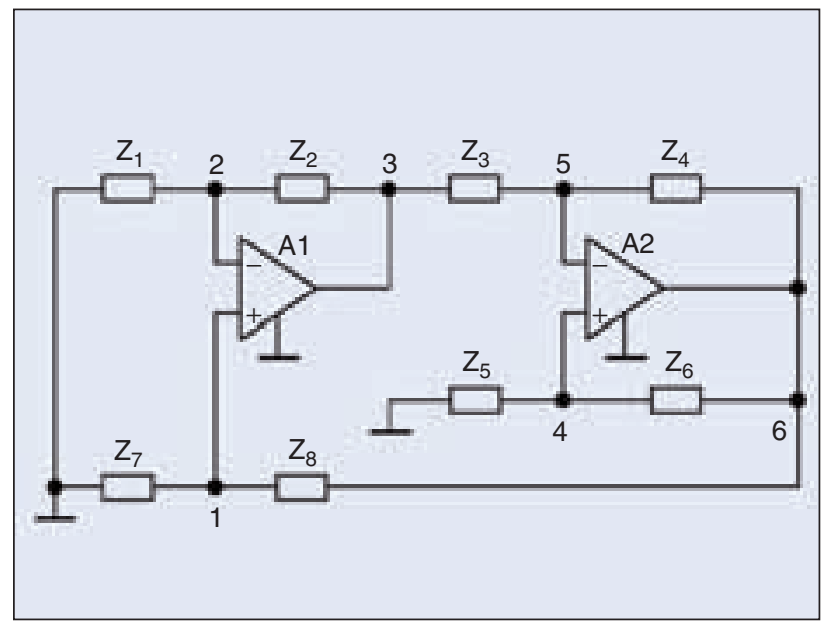

4. Quadrature oscillator. 
The quadrature oscillator is made from three circuits. For the first circuit $\left(Z_{1}, Z_{2}\right.$, and $\left.A_{1}\right)$ you have

$$
\frac{V_{3}}{V_{1}}=\frac{1+\frac{R_{2}}{R_{1}}}{1+\frac{1}{A_{1}}\left(1+\frac{R_{2}}{R_{1}}\right)} .
$$

For the second circuit $\left(Z_{3}-Z_{6}\right.$ and $\left.A_{2}\right)$, you have:

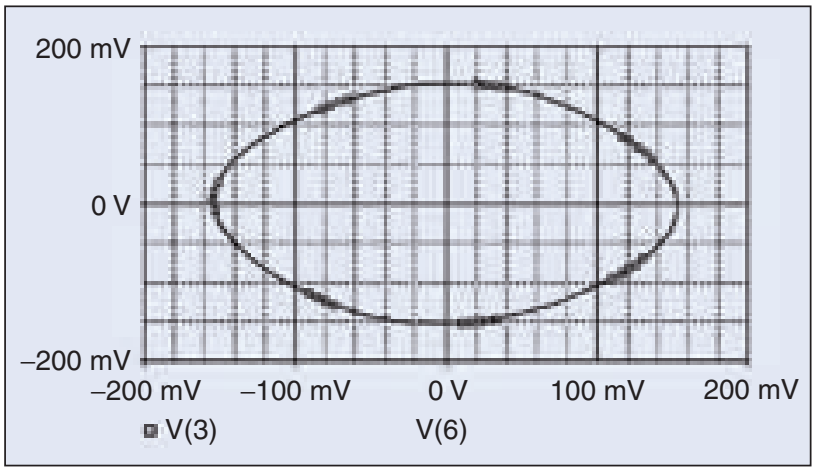

5. Quadrature oscillator. V(3) as function of $V(6)$

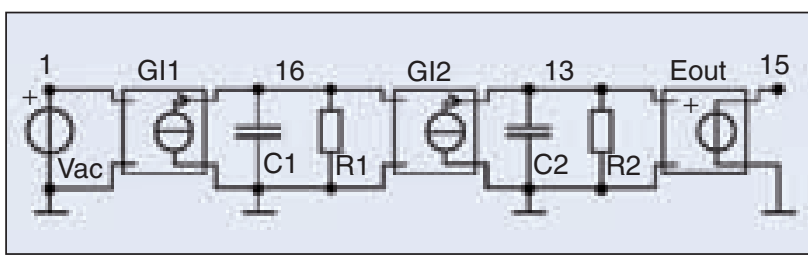

6. Op amp macro model.

$E_{\text {out }}=A(s)$ Vac $, \omega_{1}=197.77, \omega_{2}=68.375 \mathrm{M}$.

$$
\frac{V_{6}}{V_{3}}=\frac{1}{\left(1+\frac{R_{3}}{R_{4}}\right)\left(\frac{R_{5}}{R_{5}+R_{6}}-\frac{1}{A_{2}}\right)-\frac{R_{3}}{R_{4}}} .
$$

The third circuit is the feedback circuit $\left(Z_{7}\right.$ and $\left.Z_{8}\right)$ for which:

$$
\frac{V_{1}}{V_{6}}=\frac{R_{7}}{R_{7}+R_{8}} .
$$

The loop gain is the product of the three network functions.

With reference to [7], you may now design the oscillator. With $R_{1}=R_{4}=R_{7}=10 \mathrm{k} \Omega$, you get $R_{2}=33.5 \mathrm{k} \Omega$, $R_{3}=R_{8}=3 \mathrm{k} \Omega, R_{5}=1 \Omega$, and $R_{6}=1,130 \Omega$. Figure 5 shows the result of a PSpice analysis with two $\mu \mathrm{A} 741$ op amps. The frequency is $530 \mathrm{kHz}$, and the output amplitude of any of the two op amps is $155 \mathrm{mV}$.

In the PSpice macro model of the op amp, diodes and transistors are used for modelling of the nonlinear loss and memory mechanisms which give rise to a time varying operating point. Figure 6 shows a simple macro model for the op amp with two negative real poles. The model is based on the assumption of a time invariant operating point. Figures 7 and 8 show the result of an optimization of the simple model with only one nonlinear element: the piecewise-linear, voltagecontrolled current source GI1. The component values obtained are: $C 1=71.173000 \mu \mathrm{F}, \quad R 1=446.36969 \Omega$, $C 2=205.87424 p \mathrm{~F}$, and $R 2=446.35600 \Omega$, corresponding to a dominant pole at $31.48 \mathrm{HZ}$ and a $\mathrm{HF}$ pole at $10.88 \mathrm{MHz}$.

Figures 7 and 8 show the close agreement between the output voltage $V(3)$ of the PSPICE library macro model and the output

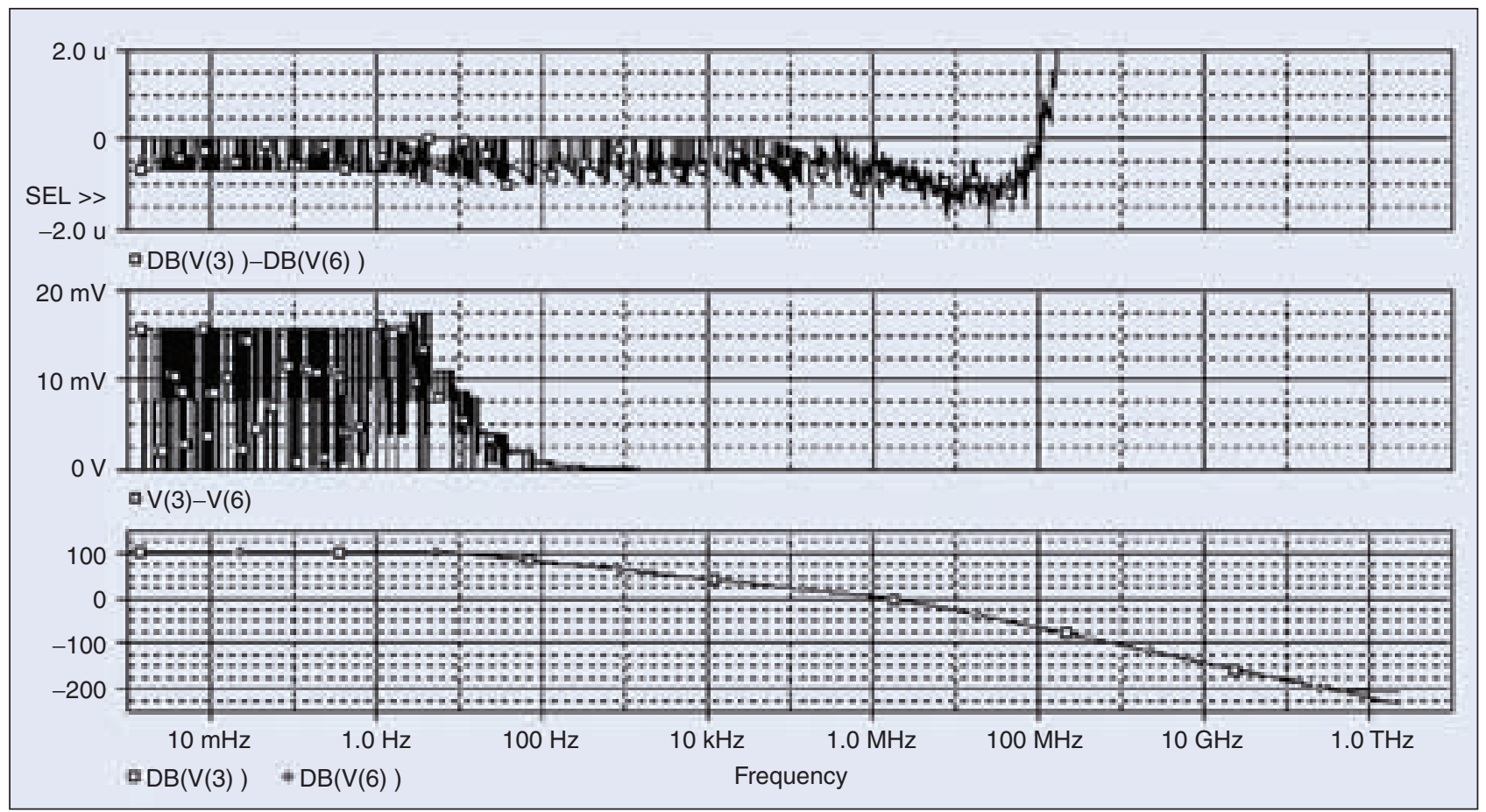

7. Comparison of the open loop ac-transfer characteristics of the op amp macro model of Figure 6 and the PSpice $\mu A 741$ library macro model. Amplitude characteristic. Logarithmic frequency scale. 


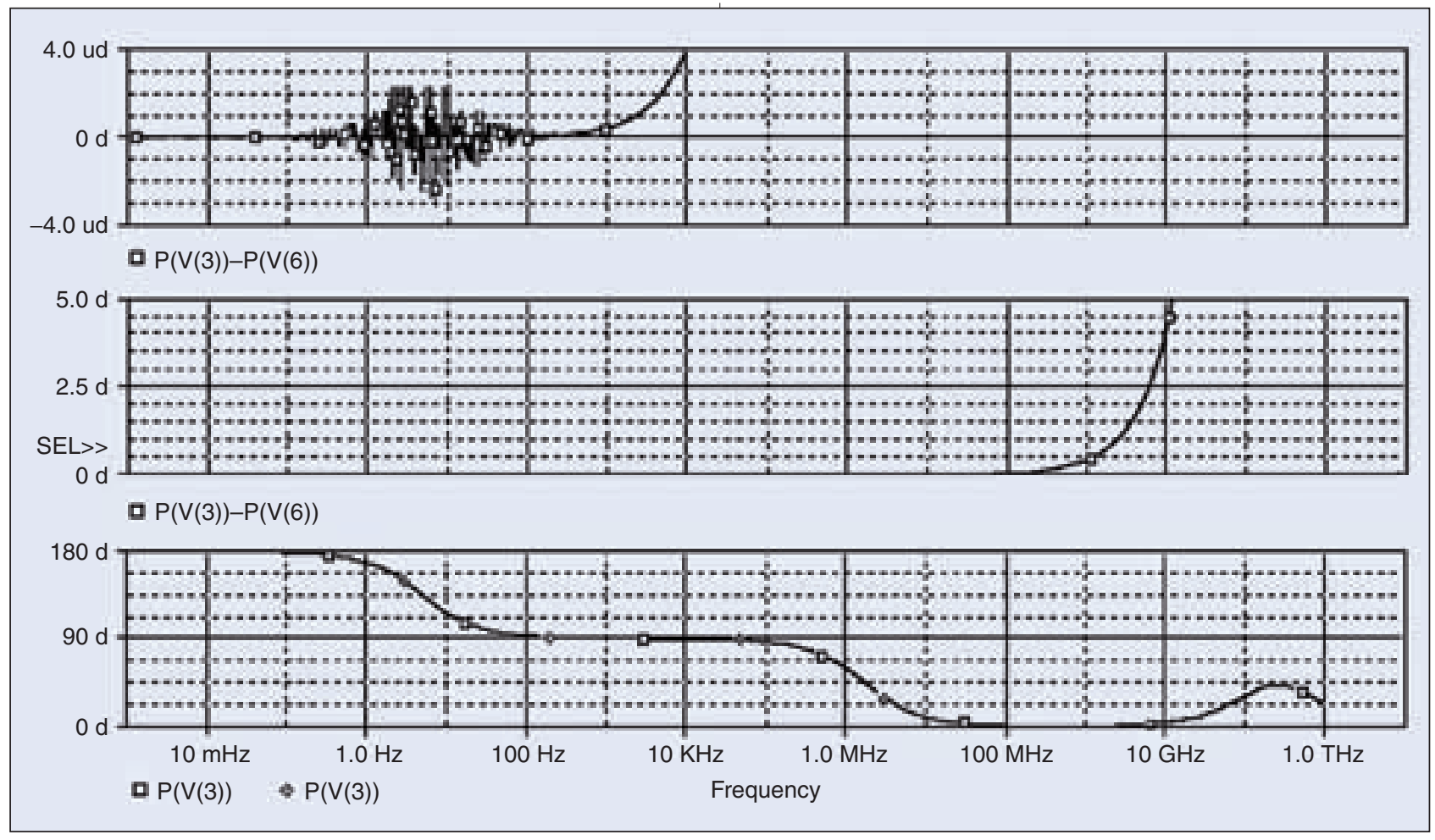

8. Comparison of the open loop ac-transfer characteristics of the operatging amperage macro model of Figure 6 and the PSpice $\mu$ A741 library macro model. Phase characteristic. Logarithmic frequency scale.

voltage $V(6)$ of the macro model in Figure 6 in the frequency range $1 \mathrm{mHz}$ to 1 THz. Figures 9 and 10 show that the hysteresis of the PSpice library macro model is not modeled in Figure 6.

There are only two nonlinear components, GI1A1 and GI1A2, in the quadrature oscillator model in Figure 6 when the simple op amp model of Figure 6 is used. These nonlinearities are piecewise linear. There are four combinations of large gain and zero gain, so you may calculate the poles as follows. For small signals, i.e., when the op amp input voltages $V(1,2)$ and $V(4,5)$ are both in the range from $-47 \mu \mathrm{V}$ to $+47 \mu \mathrm{V}$, there are two complex pole

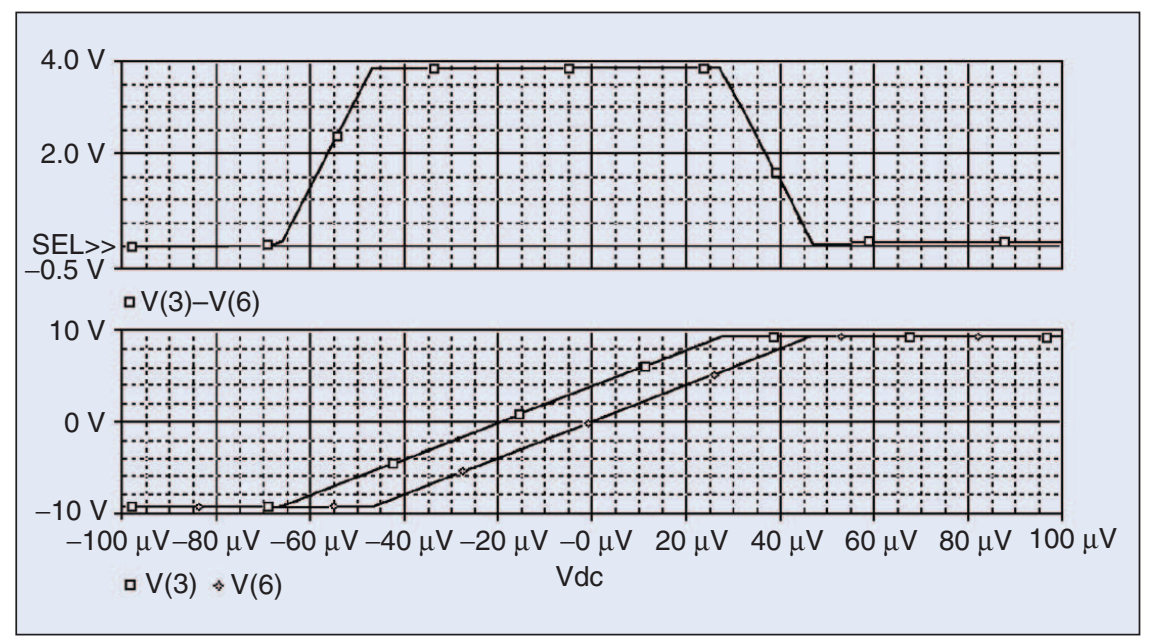

9. Comparison of the dc-transfer characteristics of the op amp macro model of Figure 6 and the PSpice library macro model. VDC is varied from $-100 \mu \mathrm{V}$ to $+100 \mu \mathrm{V}$. pairs, one in RHP and one in LHP:

\begin{tabular}{ccccc} 
real & $\pm j$ imag & real & $\pm j$ & imag \\
\hline$+400 \mathrm{k}$ & $4.49 \mathrm{M}$ & $-11.3 \mathrm{M}$ & $4.49 \mathrm{M}$ \\
\hline
\end{tabular}

When $V(1,2)$ is outside the range from $-47 \mu \mathrm{V}$ to $+47 \mu \mathrm{V}$, and $V(4,5)$ is in the range from $-47 \mu \mathrm{V}$ to $+47 \mu \mathrm{V}$, there are four negative real poles:

\begin{tabular}{cccc} 
real & real & real & real \\
\hline-31.5 & $-1.71 \mathrm{M}$ & $-9.17 \mathrm{M}$ & $-10.9 \mathrm{M}$ \\
\hline
\end{tabular}

With $V(1,2)$ in the range from $-47 \mu \mathrm{V}$ to $+47 \mu \mathrm{V}$, and $V(4,5)$ outside the range from $-47 \mu \mathrm{V}$ to $+47 \mu \mathrm{V}$, there are four negative real poles:

\begin{tabular}{cccc} 
real & real & real & real \\
\hline-31.5 & $-1.71 \mathrm{M}$ & $-9.17 \mathrm{M}$ & $-10.9 \mathrm{M}$ \\
\hline
\end{tabular}

When $V(1,2)$ and $V(4,5)$ are both outside the range from $-47 \mu \mathrm{V}$ to $+47 \mu \mathrm{V}$, there are two real double poles in LHP: 


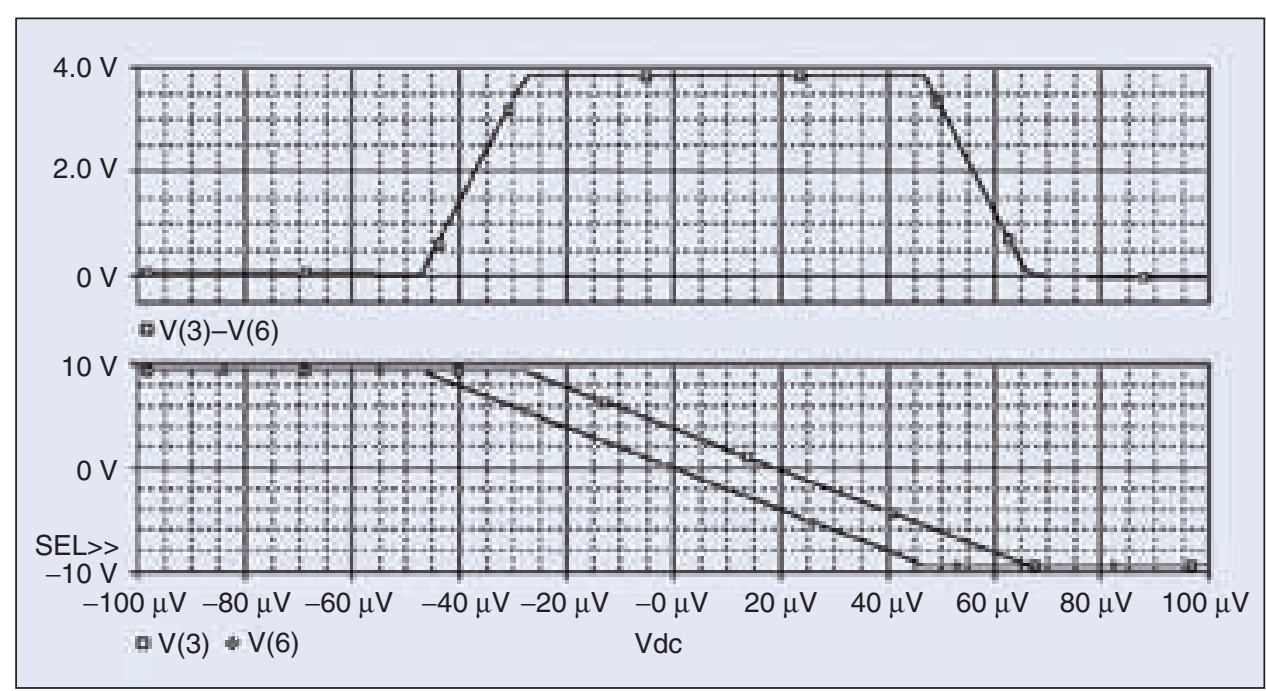

10. Comparison of the dc-transfer characteristics of the op amp macro model of Figure 6 and the PSpice library macro model. VDC is varied from $-100 \mu \mathrm{V}$ to $+100 \mu \mathrm{V}$.

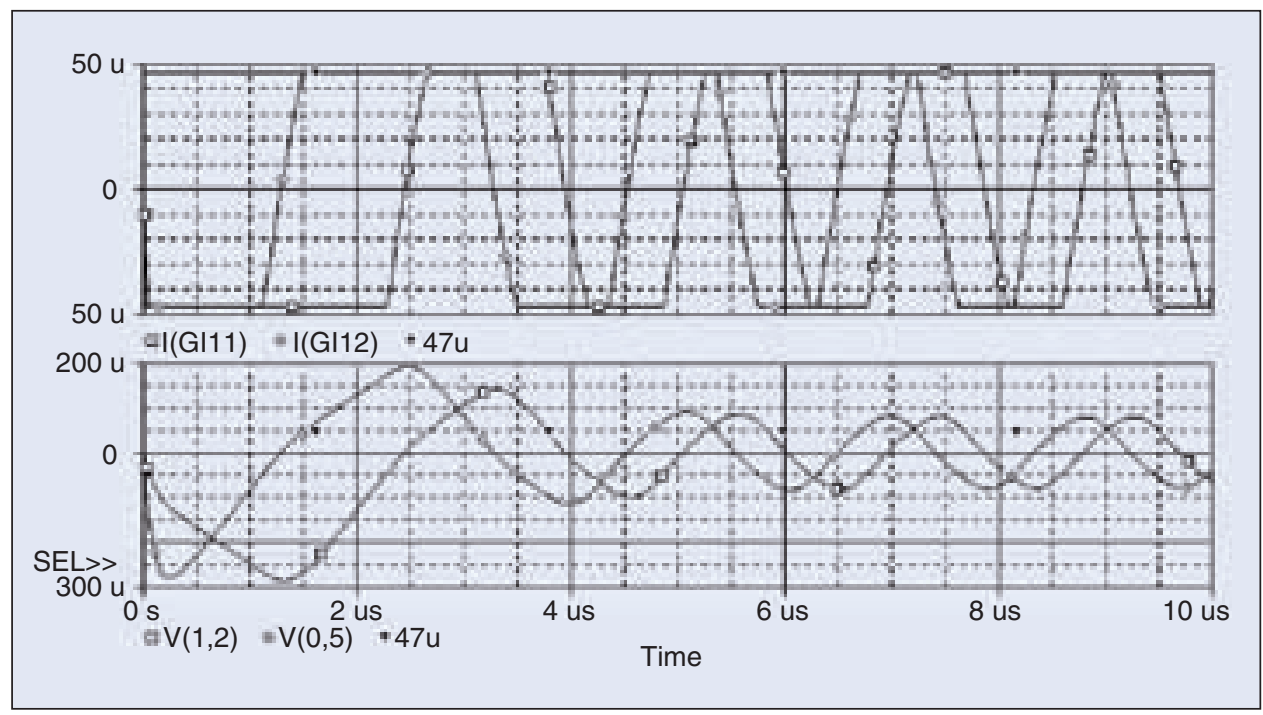

11. Input voltages of op amps (V(1,2), V(4,5)) and nonlinear currents of op amps (GI1, GI2) as functions of time (initial transient to steady state). The op amp macro model of Figure 6 used. books and replace statements concerning "nonlinearities pulling the poles back to the imaginary axis" with proper statements concerning the mechanisms behind steadystate oscillations, e.g., sinusoidal oscillation, where a complex pole pair is moving between RHP and LHP, or relaxation oscillation, where real poles are moving between RHP and LHP. The mechanism behind the behavior of a quadrature oscillator where only in the unstable dc bias point a complex pole pair occur in RHP is investigated. In the steady state, all poles are real and negative, and relaxation oscillations similar to the multivibrator performance occur. The "frozen eigenvalues" might be the starting point for a proper explanation of the mechanisms behind "phase noise."

\section{REFERENCES}

[1] E. Lindberg, K. Murali, and A. Tamasevicius (2001). "Design of chaotic oscillators," presented at ECCTD'01-the 15th European Conference on Circuit Theory and Design, Helsinki, Tutorial T5 [Online]. Available: http://www.ecs. dtu.dk/tutor-01.htm.

[2] E. Lindberg, "Colpitts, eigenvalues and chaos," in Proc. NDES'97-the 5th International Specialist Workshop on Nonlinear Dynamics of Electronic Systems, Moscow, 1997, pp. 262-267.

\begin{tabular}{cccc} 
real & real & real & real \\
\hline$-10.9 \mathrm{M}$ & $-10.9 \mathrm{M}$ & -31.5 & -31.5 \\
\hline
\end{tabular}

Only in the case where the input voltages of A1 and A2 are both small signals, a complex pole pair in RHP is present corresponding to the unstable $\mathrm{dc}$ bias point. In the other cases, the poles are real and negative, and the circuit is damped. This is in agreement with Figure 11. It is seen how the two op amps synchronize so that only one is active at a time. A certain amount of energy is moving between the memory elements corresponding to the input level of $47 \mu \mathrm{V}$ above which the gain is zero.

\section{CONCLUSIONS}

This article demonstrated that it is possible to obtain insight in the mechanisms behind the behavior of oscillators by means of "the frozen eigenvalues approach." We must rewrite our text-
[3] E. Lindberg, "Oscillators and eigenvalues," in Proc. ECCTD'97-The 1997 European Conference on Circuit Theory and Design, Budapest, 1997, pp. 171-176.

[4] J.R. Westra, C.J.M. Verhoeven, and A.H.M. van Roermund, Oscillators and Oscillator Systems-Classification, Analysis and Synthesis. Norwell, MA: Kluwer 1999, pp. 1-282.

[5] A.S. Sedra and K.C. Smith, Microelectronic Circuits, 4th ed. London, U.K.: Oxford Univ. Press, 1998.

[6] L. Strauss, Wave Generation and Shaping. New York: McGraw-Hill, 1960, pp. 1-520.

[7] E. Vidal, A. Poveda, and M. Ismail, "Describing functions and oscillators," IEEE Circuits Devices Mag., vol. 17, pp. 7-11, Nov. 2001.

Erik Lindberg is with the Technical University of Denmark in Kongens Lyngby, Denmark. E-mail: el@oersted.dtu.dk or erik.lindberg@ieee.org. 